\title{
The veterinary anti-parasitic selamectin is a novel inhibitor of the mycobacterial DprE1 enzyme
}

José Manuel Ezquerra-Aznárez 1, Giulia Degiacomi 2, Henrich Gašparovič 3, Giovanni Stelitano ${ }^{2}$, José Camilla Sammartino ${ }^{2}$, Jana Korduláková ${ }^{3}$, Paolo Governa ${ }^{4}$, Fabrizio Manetti 4, Maria Rosalia Pasca ${ }^{2}$, Laurent Roberto Chiarelli2, "* and Santiago Ramón-García 1, 5, 6, *

1 Department of Microbiology, Faculty of Medicine, University of Zaragoza, Spain

2 Department of Biology and Biotechnology "Lazzaro Spallanzani", University of Pavia, Italy

3 Department of Biochemistry, Faculty of Natural Sciences, Comenius University in Bratislava, Slovakia

4 Department of Biotechnology, Chemistry and Pharmacy, University of Siena, Italy

5 Research \& Development Agency of Aragon (ARAID) Foundation, Spain

6 CIBER Enfermedades Respiratorias (CIBERES), Instituto de Salud Carlos III, Spain.

* Correspondence: santiramon@unizar.es (S. R.-G.) \& laurent.chiarelli@unipv.it (L. R. C.)

Abstract: Avermectins are macrocyclic lactones with anthelmintic activity. Recently, they were found to be effective against Mycobacterium tuberculosis, which accounts for one third of the worldwide deaths from antimicrobial resistance. However, their anti-mycobacterial mode of action remains to be elucidated. The activity of selamectin was determined against a panel of $M$. tuberculosis mutants. Two strains carrying mutations in DprE1, the decaprenylphosphoryl- $\beta$-D-ribose oxidase involved in the synthesis of mycobacterial arabinogalactan, were more susceptible to selamectin. Biochemical assays against the Mycobacterium smegmatis DprE1 protein confirmed this finding, and docking studies predicted a binding site in a loop that included Leu275. Sequence alignment revealed variants in this position among mycobacterial species, with the size and hydrophobicity of the residue correlating with their MIC values; M. smegmatis DprE1 variants carrying these point mutations validated the docking predictions. However, the correlation was not confirmed when M. smegmatis mutant strains were constructed and MIC phenotypic assays performed. Likewise, metabolic labeling of selamectin-treated M. smegmatis and M. tuberculosis cells with ${ }^{14} \mathrm{C}$-labeled acetate did not reveal the expected lipid profile associated with DprE1 inhibition. Together, our results confirm the in vitro interactions of selamectin and DprE1 but suggest that selamectin could be a multi-target anti-mycobacterial compound.

Keywords: avermectins, selamectin, tuberculosis, Mycobacterium tuberculosis, drug repurposing 


\section{Introduction}

Tuberculosis (TB) is the leading cause of death among bacterial infections. In 2019, an estimated 1.4 million people died from TB, and 10 million people developed the disease [1]. The situation is aggravated by the emergence of multidrug resistant (MDR) and extensively drug resistant (XDR) strains, which are responsible for over half a million cases every year. Up until recently, MDR/XDR-TB therapy could take up to 24 months requiring the use of second-line drugs with more severe and frequent side effects that complicate patients' adherence to the treatment. The introduction of novel classes of anti-tubercular agents over the last decade has allowed for the development of new more effective regimes to treat MDR- and XDR-TB [2-4]. However, the overall success rate of drug-resistant TB therapies remains low, as only $50 \%$ of MDR-TB and one third of XDR-TB cases have positive outcomes [1,5]. Drugresistant TB is still the first cause of death due to antimicrobial resistance [6], and the World Health Organization establishes Mycobacterium tuberculosis, the causative agent of $\mathrm{TB}$, as one of the top priorities for the development of new antimicrobials [7].

Drug repurposing, i.e. finding new applications for clinically approved drugs, is an alternative to the traditionally time and resource consuming drug discovery process [8]. Following this approach, the anti-parasitic family of the avermectins was found to kill $M$. tuberculosis and other mycobacteria, a surprising finding since they were traditionally believed to be inactive against bacteria [9-11]. Avermectins are a family of 16-membered macrocyclic lactones with broad spectrum anthelmintic activity initially isolated from the soil-dwelling Streptomyces avermitilis [12]. In humans, ivermectin has been used for over three decades in the prevention and control of onchocerciasis and lymphatic filariasis [13], and it is now proposed as part of novel strategies for malaria vector control [14]. Other members of the family are also widely used to treat endo and ectoparasite infections in livestock and pets [15]. Selamectin is a veterinary avermectin used in cats and dogs with improved pharmacokinetic properties and lower toxicity over other avermectins [10]. Selamectin is the avermectin of choice for Border Collie dogs, a breed that is highly susceptible to avermectins because of the lack of a functional P-glycoprotein at the blood-brain barrier level. In addition, it shows better potency that other avermectins against mycobacteria $[9,16]$. The mode of action of avermectins against parasites is well-known: they bind to glutamate-gated chloride channels in nerve and muscle cells, increasing permeability to chloride ions and eventually causing death by paralysis [17]. However, their antimycobacterial mode of action remains to be elucidated.

DprE1 is a decaprenylphosphoryl- $\beta$-D-ribose (DPR) oxidase, which acts together with DprE2 to provide decaprenyl-phosphoryl- $\beta$-D-arabinose (DPA). The epimerization of DPR to DPA is achieved by the oxidation of DPR to the intermediate decaprenylphosphoryl-2-keto- $\beta$-D-erythropentofuranose (DPX) by DprE1 and the subsequent reduction of DPX to DPA by DprE2 [16]. DPA is the sole precursor for the 
synthesis of the arabinan moiety of the mycolyl-arabinogalactan-peptidoglycan (mAGP) complex, which is unique of mycobacteria and closely related species conferring intrinsic resistance to several antibiotics [19]. DprE1 is essential for $M$. tuberculosis growth [20], and a well-established antitubercular drug target, being inhibited by more than 15 different pharmacophores including the family of benzothiazinones, currently in Phase II clinical trials [5,21,22].

In this work, we identified DprE1 as a possible target of avermectins in mycobacteria and characterized this activity using selamectin as a model avermectin. We found that selamectin is able to inhibit the activity of the DprE1 enzyme, binding it through hydrophobic interactions that involve the residue at position 282 in $M$. smegmatis (corresponding to $M$. tuberculosis DprE1 residue 275). However, lack of phenotypic translation of these findings suggests a multi-target activity of selamectin against mycobacteria.

\section{Results}

2.1. Mycobacterium tuberculosis DprE1 mutants are more susceptible to selamectin

The Minimal Inhibitory Concentration (MIC) of selamectin was determined against a set of $M$. tuberculosis harboring different known mutations in genes encoding for drug targets (NTB1, DR1, 88.7), drug activators (53.3 and 81.10), or drug inactivator (Ty1) (Table 1). Resistance to selamectin was not observed; however, NTB1 and NTB9 strains displayed a consistent two-fold increase in sensitivity to the compound. These strains carry the mutations C387S and C387G in DprE1, respectively, conferring highlevel resistance to benzothiazinones [22]. By contrast, the overexpression of $d p r E 1$ gene (H37Rv-pSODIT-2:dprE1 strain) did not confer changes in the susceptibility to selamectin (Table 1).

Time-kill kinetics assays were performed to confirm the increase in susceptibility of NTB1 M. tuberculosis mutant to selamectin, compared to the reference parental H37Rv strain. Selamectin was more active against NTB1 as illustrated by a marked CFU drop at earlier time-points. While the limit of detection (1-log CFU/mL) was reached at day 11 when treating the NTB1 strain, this was only reached at day 22 in the case of the H37Rv control strain. In addition, lower concentrations ( $8 \mu \mathrm{g} / \mathrm{mL})$ of selamectin were more active against the NTB1 strain compared to the control strain (Figure 1).

\subsection{Avermectins inhibit the DprE1 enzyme in vitro}

Two M. tuberculosis strains carrying mutations in DprE1 were more sensitive to selamectin (Table 1). To evaluate whether this enzyme could be involved in the mechanism of action of avermectins, the in-vitro effects of the compounds were assessed against the DprE1 enzyme. Four avermectins were thus tested against the 
purified Mycobacterium smegmatis DprE1 recombinant enzyme. All of them were significantly active against DprE1, being selamectin the best inhibitor with an IC50 of $2.6 \pm 1.1 \mu \mathrm{M}$ (Table 2).

The two M. tuberculosis NTB1 (C387S) and NTB9 (C387G) mutant strains carry a mutation in the Cys387 residue of the DprE1 enzyme, known to be responsible for the binding to BTZ-043 [22]. To clarify the role of this residue in the inhibition by avermectins, the corresponding Cys394 in the M. smegmatis DprE1 enzyme was mutated to Ser (C394S), Gly (C394G) and Ala (C394A), the enzymes produced and purified. Mutation C394A was also investigated since all mycobacteria harboring this residue are resistant to BTZ [22].

The activity of the C394G mutant enzyme was negligible, as already reported [23], while C394S and C394A were active, but showed impaired kinetic parameters, leading to about 5 -fold lowered specificity constants with respect to the wild-type (Table 3). Selamectin was able to bind both mutant enzymes, showing $\mathrm{IC}_{50}$ in the same range (C394A) or slightly higher (C394S) than the wild type enzyme. This suggests that, although avermectins inhibit the activity of DprE1 (Table 2), binding to the DprE1 active site is not primary related to the cysteine residue, similarly to other non-covalent inhibitors [24,25].

\subsection{In silico model of selamectin-DprE1 binding}

Evidence above described showed a secondary role of the M. smegmatis DprE1 Cys394 residue in the mechanism of inhibition of selamectin. Thus, to investigate alternative DprE1 binding sites of selamectin an in silico approach was used with a representative set of selamectin conformations docked into the structure of DprE1. Due to its high molecular size, selamectin was unable to fully penetrate the DprE1 binding site only occupying a portion of the ligand binding site close to the FAD cofactor, which accom-modated the co-crystalized quinoxaline inhibitors of the $X$-ray crystallographic structures [25]. A large portion of the selamectin structure occupied a tunnel pointing toward the enzyme surface. An analysis of the best-scored binding poses showed that a hydrogen bond was formed between the oxime nitrogen atom and the guanidino moiety of the Arg325 side chain (Figure 2). The same amino acid is involved in a hydrogen bond with the nitro group of the quinoxaline inhibitor QN129 in complex with DprE1 (PDB 4P8T) (Figure S1). Moreover, part of both spiro and condensed heterocycles of selamectin were within the region occupied by the alkoxy-propanol ligand present in the complex [25]. Finally, the oxygen atom of the methoxy group of selamectin made an additional hy-drogen bond with the hydroxyl substituent of the Tyr297 side chain, close to the sol-vent-exposed protein surface. It is worth noting that the lateral wall of the cavity included Leu275.

Molecular dynamics simulations were also performed to evaluate the timedependent stability of the selamectin-DprE1 complex. The evolution of the root-mean 
square deviation of 3D coordinates of selamectin atoms during MD simulation time in comparison to the last frame of minimization showed a $1.28 \AA$ mean value with a 0.22 standard deviation, thus suggesting a significant stability of the complex. Moreover, a cluster analysis of the MD production phases aimed at evaluating the most frequent interactions of selamectin confirmed the hydrogen bonds with both Arg325 and Tyr297.

Even if the primary sequence of DprE1 belonging to different mycobacteria is conserved in the region that constitutes the binding site, sequence alignment revealed Leu275 polymorphisms in different species: $M$. avium carries a Val; M. bovis, $M$. tuberculosis, M. smegmatis and M. abscessus, a Leu; and M. kansasii, a Phe (Figure 3 and Figure S2). Increasing the bulkiness and lipophilicity of the amino acid chain from an isopropyl group of Val to an isobutyl chain of Leu to a benzyl moiety of Phe could enhance the interaction between selamectin and DprE1, therefore increasing the susceptibility of the enzyme to the ligand. In the last case, the aromatic side chain of Phe275 would be accommodated in a region of space that contains a cage of additional aromatic residues, such as Phe279, Phe320, and Trp323. The interactions between selamectin and DprE1 could be facilitated by the presence of Phe275 that enhances the hydrophobic contacts close to the binding site when compared to Val and Leu. Thus, such aromatic machinery could be responsible for the stabilization of the interaction between selamectin and DprE1.

2.4. Position Leu282 is involved in the binding of selamectin to DprE1 but lacks molecular to phenoty pic translation

To verify our hypothesis about the binding of selamectin to DprE1, two $M$. smegmatis DprE1 mutant enzymes were constructed introducing a Val or a Phe at the position 282 (corresponding to M. tuberculosis DprE1 residue 275). The introduction of these mutations did not affect the catalytic activity of the enzyme since the kinetic parameters of the mutants were similar to those of the wild-type DprE1 (Table 3). By contrast, they displayed different responses to selamectin; the L282F mutant displayed an IC50 ca. 2-fold lower and the L282V mutant an IC50 ca. 20-fold higher than that of the wild-type (Table 3 and Figure 4). These data validated the in silico predictions and confirmed the importance of this residue at position 282 to stabilize the binding of selamectin to DprE1 through hydrophobic interactions.

To ascertain whether the antimycobacterial activity of selamectin relies in DprE1 inhibition, the $\mathrm{L} 282 \mathrm{~F}$ and $\mathrm{L} 282 \mathrm{~V}$ mutations were then introduced in $M$. smegmatis DprE1 using single-stranded DNA recombineering to assess the molecular to phenotypic translation of selamectin activity (Figure S3). MIC assays were performed against the mutant strains with no changes in their susceptibilities to selamectin compared to wild-type $M$. smegmatis (Table 4); these observations were also confirmed by time-kill kinetic assay (Figure S4). 
The effects of selamectin on the mycobacterial lipid composition were also investigated by metabolic labeling. Treatment of M. tuberculosis H37Rv or M. smegmatis $\mathrm{mc}^{2} 155$ cells with selamectin at final concentrations of 4,8 and $20 \mu \mathrm{g} / \mathrm{mL}$ or $4,8,16$ and $40 \mu \mathrm{g} / \mathrm{mL}$, respectively, did not result in significant changes in the lipid composition associated with the inhibition of arabinan synthesis. A general decrease in ${ }^{14} \mathrm{C}$ uptake was observed upon selamectin treatment (Figure 5).

Altogether, these data suggest that, although the enzyme is efficiently inhibited by selamectin, DprE1 might not be the primary target and supports the hypothesis of avermectins being multi-targeting compounds.

\section{Discussion}

Drug repurposing is gaining traction across the pharmaceutical industry, given its potential advantages over the de novo drug discovery programs [9] in terms of time and economic investments. Drug repurposing is especially relevant in the case of underfunded diseases, such as tuberculosis, or neglected diseases where revenues are not expected to compensate the investment effort. Moreover, antimicrobials in general are also perceived as less profitable than other drugs because they are used to treat acute diseases and, since their lifespan is jeopardized by the emergence of drug resistant strains, new antibiotics are kept as last-resort options to preserve their activity. Indeed, repurposing efforts have already broadened our armamentarium to treat MDR- and XDR-TB, such as linezolid or carbapenems [2,5].

Linezolid and carbapenems target structures that are highly conserved amongst bacteria (the ribosome and penicillin-binding proteins) and thus, their mode of action can be assumed to be similar across bacteria. However, this is not the case for avermectins; although their mode of action has been defined for helminths [27] it is unlikely that it translates into mycobacteria. Thus, to get insight into the mechanism of action of selamectin, we screened a panel of $M$. tuberculosis strains with wellcharacterized mechanisms of resistance to a variety of avermectin molecules. We found that, while none of the mutations conferred resistance to selamectin, mutant strains NTB1 and NTB9 with C387S and C387G mutations in DprE1, respectively, were more susceptible (Table 1), suggesting a potential role for DprE1 in the mode of action of selamectin.

DprE1 is a promising target for the development of new anti-tubercular drugs, with chemical scaffolds targeting this enzyme currently moving into the clinical studies [21]. Thus, we delved into the potential interaction between the avermectins and DprE1 by testing their activity against the purified enzyme. Our data confirmed that avermectins inhibit DprE1 in vitro, although with lower potency than benzothiazinones, being selamectin the most active with an IC50 of ca. $2.6 \mu \mathrm{M}$ (Table 2). In addition, the mutant enzymes carrying the mutations present in the selamectin susceptible NTB1 (C387S) M. tuberculosis strain (which confer high-level resistance to 
BTZ043) and, especially, the mutation harboring an Ala residue in this position (C387A) showed selamectin IC50 values slightly different to that of the wild-type (Table 3). It is noteworthy that all the mutant enzymes displayed significantly altered catalytic properties. In the light of these results, we could hypothesize that the phenotypic effect observed in the NTB1 and NTB9 $M$. tuberculosis strains is the result of a reduced catalytic efficiency displayed by the mutant DprE1 forms, which would render $M$. tuberculosis more vulnerable to other chemical interactions with DprE1. Thus, lower concentrations of selamectin would suffice for activity below a critical threshold, as demonstrated by time-kill kinetic assays (Figure 1).

Cysteine 387 is essential for the activity of covalent inhibitor of DprE1, such as benzothiazinones, but usually this residue is not the primary interaction with reversible inhibitors; this is the case of avermectins. Thus, to investigate other possible binding sites of selamectin, we performed in silico studies. Docking revealed a potential site in a cavity where known compounds block DprE1 through non-covalent inhibition [25]. Protein sequence in this region of the DprE1 enzyme was highly conserved among different mycobacteria (Figure S2) and shows almost no variation except for Leu275. Our finding that the moiety at the position 275 influences selamectin binding to DprE1 was consistent with the previous reports of selamectin activity against mycobacteria: $M$. avium, which carries a Val residue (with the smallest side chain in the series) at the 275 position is the less sensitive $(\mathrm{MIC}=16 \mu \mathrm{g} / \mathrm{mL}$ ); $M$. bovis, $M$. tuberculosis and $M$. smegmatis carry a Leu and show a medium sensitivity to selamectin (MIC $=2-4 \mu \mathrm{g} / \mathrm{mL}$ ); M. kansasii, bearing the largest amino acid (Phe) is the most sensitive to selamectin $(\mathrm{MIC}=0.5 \mu \mathrm{g} / \mathrm{mL})[9,11]$. M. abscessus, carrying a Leu, represents an exception with an MIC $>32 \mu \mathrm{g} / \mathrm{mL}$ [11]; however, M. abscessus DprE1 carries an extra N-terminal 40 residues domain and shows the lowest sequence conservation (Figure S2).

To fully discern the actual role of the different amino acids at position 275 (282 in the $M$. smegmatis sequence) in the anti-mycobacterial activity of selamectin, we performed biochemical and genetic studies with M. smegmatis. Firstly, we produced the recombinant DprE1 mutant proteins in which the Leu residue at position 282 was replaced by Val and Phe. Both enzymes showed differences in their affinities to selamectin, demonstrating that this residue is involved in hydrophobic interactions with the compound. Indeed, the substitution of the Leu with the small Val led to a significantly lowered (20-fold) affinity to selamectin, indicating that this residue is unable to correctly interact with the compound. This was confirmed by the introduction of the Phe residue, which led to a 3 -fold decrease in the $\mathrm{IC}_{50}$, confirming that a bulkier and more hydrophobic residue stabilizes the binding (Table 3).

Nevertheless, when the same mutations were introduced in the M. smegmatis genome, these did not translate into differences in the susceptibility to selamectin (Table 4). Moreover, despite the good activity of the compound against the in vitro enzyme activity, treatment with selamectin did not lead to alterations in the cell wall 
composition typical of DprE1 inhibition. Indeed, compounds inhibiting DprE1 activity in mycobacterial cells block the synthesis of the cell wall arabinan. The decrease of an arabinan acceptor for mycolic acids attachment leads to diminished amount of cell wall bound mycolates and accumulation of mycolic acids in the forms of trehalose monomycolates and trehalose dimycolates [28]. In our case, although a general decrease in ${ }^{14} \mathrm{C}$ uptake was observed upon selamectin treatment, metabolic labeling of selamectin-treated $M$. smegmatis and $M$. tuberculosis did not show the changes in the lipid profile typically associated to the inhibition of DprE1 (Figure 5).

In this study we have demonstrated that avermectins are inhibitors of the DprE1 enzyme, highlighting the role of the amino acid at positions 275 (in $M$. tuberculosis) in stabilizing their interaction at the molecular level. This is the first time that this residue is described to have a role in the inhibition of DprE1 activity. However, lack of molecular to phenotypic translation of DprE1 inhibition indicates that DprE1 could be only one among many factors underlying selamectin activity in mycobacteria. Avermectins are conceivably not covalent inhibitors of DprE1, and show overall relatively high IC50 values, compared to other DprE1 inhibitors that display activity in the $\mathrm{nM}$ concentration range [21]. So, the potency of the interactions might be too low to translate the in vitro activity into differential growth inhibition, concealing the effect of inhibition under normal growth conditions. Indeed, M. tuberculosis NTB1 and NTB9 mutant strains (with a DprE1 enzyme highly compromised in their catalytic efficiency) are more sensitive to avermectins. In this case the intrinsically lower activity of the mutant enzymes could result inadequate to sustain bacterial viability in the presence of inhibitors at a certain concentration range (Figure 1).

Because the avermectins have a clear phenotypic inhibitory activity against mycobacteria (with MIC values in the low $\mu \mathrm{M}$ range) these data strongly suggest alternative DprE1-independent molecular target(s). The multi-targeting nature of the avermectins might not come to a surprise considering not only their anthelmintic properties, but also antimicrobial, antiviral, antifungal, as well as anticancer and antidiabetic activities [29]. In this context multi-targeting or poly-pharmacology approaches to develop new anti-tubercular drugs are gaining interest among the drug developers. Indeed, the use of a single molecule acting on different targets has several benefits, such as possible lower cytotoxicity, lower rate of resistance development, and higher therapeutic efficacy, compared to the classical approach [30]. Together with the future identification of other mycobacterial targets, our finding paves the way for a rational design to improve their potency against DprE1 and develop a new generation of avermectins.

\section{Materials and methods}

$\underline{4.1 . B a c t e r i a l ~ s t r a i n s, ~ r e a g e n t s ~ a n d ~ c u l t u r e ~ m e d i a ~}$ 
Mycobacterium smegmatis and Mycobacterium tuberculosis were routinely grown in Middlebrook 7H9 broth (Difco) supplemented with 10\% Middlebrook Oleic acidAlbumin-Dextrose-Catalase (OADC) or ADC (Difco) and $0.05 \%(\mathrm{v} / \mathrm{v})$ Tyloxapol or $0.025 \%$ Tween80 (v/v) or on Middlebrook 7H10 or 7H11 agar supplemented with $10 \%$ (v/v) OADC (Difco). For plasmid-carrying strains, hygromycin B (Invivogen) was added at $20 \mu \mathrm{g} / \mathrm{mL}$. Ivermectin, milbemycin oxime, moxidectin and selamectin were purchased from the European Pharmacopoeia.

\subsection{Drug susceptibility testing}

Two-fold serial dilutions of the compounds were prepared in 7H9 broth supplemented with $0.2 \%$ glycerol and 10\% ADC in 96-well flat-bottomed plates and seeded with $M$. smegmatis at a bacterial density of $10^{5} \mathrm{CFU} / \mathrm{mL}$. Plates were incubated for 3 hours before addition of $30 \mu \mathrm{L}$ of the bacterial growth reporter MTT [3-(4,5dimethylthiazol-2-yl)-2,5-diphenyltetrazolium bromide] $(2.5 \mathrm{mg} / \mathrm{mL}$ ) (Sigma) and Tween $80(10 \%, v / v)$ (Scharlau). The optical density at $580 \mathrm{~nm}$ was measured after further 3-hour incubation, the lowest compound concentration that inhibited $90 \%$ MTT conversion to formazan was used to define the MIC value.

M. tuberculosis strains were tested in duplicate in 96-well flat-bottomed plates in $7 \mathrm{H} 9$ with $10 \%$ OADC in a final volume of $200 \mu \mathrm{L}$. Bacteria were incubated for 7 days in the presence of the compounds before the addition of $10 \mu \mathrm{L}$ of $0.025 \mathrm{mg} / \mathrm{mL}$ resazurin. Following 1-day incubation, compound activity was determined by fluorescence measures (530 $\mathrm{nm}$ excitation, $590 \mathrm{~nm}$ emission).

\subsection{Time-kill kinetics assays}

A starting inoculum of ca. $10^{5} \mathrm{CFU} / \mathrm{mL}$ of $M$. tuberculosis was pre-incubated in $7 \mathrm{H} 9$ with $10 \%$ OADC for 3 days at $37^{\circ} \mathrm{C}$ before the addition of $0.1,0.5,2,8$ or $32 \mu \mathrm{g} / \mathrm{mL}$ of selamectin (corresponding to $0.025,0.125,0.5,2$ and 16-fold the MIC against $M$. tuberculosis H37Rv). Then 10-fold serial dilutions were plated onto 7H11 agar with $10 \%$ OADC at $0,1,3,7,11,14$ and 22 days after compound addition, and CFUs enumerated after three weeks of incubation at $37^{\circ} \mathrm{C}$.

For M. smegmatis, $10 \mathrm{~mL}$ of $7 \mathrm{H} 9$ with $0.2 \%$ glycerol (v/v) and $10 \%$ ADC were inoculated with $M$. smegmatis $\mathrm{mc}^{2} 155$ cells from liquid cultures in late exponential phase to a final density of $10^{5} \mathrm{CFU} / \mathrm{mL}$. Cultures were grown at $37{ }^{\circ} \mathrm{C}$ in the presence of $4 \mu \mathrm{g} / \mathrm{mL}$ and $16 \mu \mathrm{g} / \mathrm{mL}$ of selamectin (1 or 4 -fold the compound MIC against the wildtype $M$. smegmatis) and $4 \mu \mathrm{g} / \mathrm{mL}$ and $16 \mathrm{ng} / \mathrm{mL}(0.5$ or 2 -fold the compound MIC against the wild-type $M$. smegmatis) and aliquots (100 $\mu \mathrm{L})$ taken at 0, 24, 48, 72 and 96 hours post inoculation. Then, 10 -fold serial dilutions were seeded onto Luria-Bertani (LB) agar plates and CFUs enumerated after 3 days of incubation at $37^{\circ} \mathrm{C}$.

\subsection{Docking of selamectin to DprE1 and molecular dynamics simulations}


Twenty-nine three-dimensional structures of unbound or complexed DprE1 from $M$. tuberculosis or $M$. smegmatis, obtained by means of X-ray crystallographic experiments and characterized by a resolution ranging from 1.79 to $3 \AA$, are available within the Protein Data Bank. However, while the coordinates of 4P8L (sequence Gly5Leu461, $2.02 \AA$ resolution) and 4P8T (sequence Ala6-Leu461, $2.55 \AA$ resolution) are completely solved [26], the remaining structures showed unsolved regions. Although with slightly lower resolution, 4P8T was used for computer simulations because it accommodated a non-covalent quinoxaline derivative (namely, QN129) bulkier than the quinoxaline Ty36c bound to DprE1 in the complex 4P8L [26]. The threedimensional coordinates of the complex between DprE1 and the 3-[(4cyanobenzyl)amino]-6-(trifluoromethyl)quinoxaline-2-carboxylic acid QN129 (PDB 4P8T) were submitted to the Protein Preparation Wizard of the Maestro suite [31] to assign bond orders, add hydrogens to the appropriate positions, and remove water molecules. Next, the Receptor Grid Generation routine was applied to the complex with the aim of codifying the physico-chemical properties of amino acids that constitute the DprE1 active site. Hydroxyl groups (in Tyr and Ser residues) within the grid were free to rotate. A Monte Carlo Multiple Minimum conformational search was applied to selamectin using OPLS3e as the force field and water as the solvent. Conformers were minimized with the Polak-Ribiere conjugate gradient until convergence on movement (0.05 $\AA$ threshold). Molecular docking simulations were performed by means of Glide software [32], with standard precision and flexible ligand sampling.

Following molecular docking calculations, molecular dynamics (MD) simulations were performed to check for the stability of the best-ranked docking complex using the Desmond software [33]. Before simulations, docked complex was solvated in a box of explicit water molecules (a TIP3P explicit solvent model) containing counterions to neutralize the charge. The overall system was then relaxed by energy minimization, heated to $300 \mathrm{~K}$, and equilibrated to finally produce a $100 \mathrm{~ns}$ MD trajectory. Default settings were used for all other parameters.

\subsection{DprE1 enzymatic studies}

Recombinant M. smegmatis DprE1 was expressed in E. coli and purified as previously reported [21]. Mutants were generated using QuikChange site-directed mutagenesis kit, following manufacturer's recommendations with the primers reported in Table S1. Enzyme activity was determined with the Amplex Red/peroxidase coupled assay [23]. DprE1 was incubated in a reaction mixture containing $20 \mathrm{mM}$ glycylglycine ( $\mathrm{pH}$ 8.5), $500 \mu \mathrm{M}$ farnesylphosphoryl- $\beta$-Dribofuranose (FPR), $50 \mu \mathrm{M}$ Amplex Red (Sigma-Aldrich) and $0.35 \mu \mathrm{M}$ horseradish peroxidase. The formation of resorufin was followed at $560 \mathrm{~nm}\left(\varepsilon=54,000 \mathrm{M}^{-1} \mathrm{~cm}^{-1}\right)$. Steady-state kinetic parameters were determined by assaying the enzyme at different FPR concentrations, and the kinetic constants determined by fitting the data to the Hill 
equation. Initial inhibition by all four avermectins was tested at $100 \mu \mathrm{M}$, using DMSO as negative control. IC50 values were subsequently determined according to the following equation:

$$
A_{[I]}=A_{[0]} *\left(1-\frac{[I]}{[I]+I C_{50}}\right)
$$

where $A_{[l]}$ was the enzyme activity in the presence of a given concentration of inhibitor [I], and $\mathrm{A}_{[0]}$ the enzyme activity in the absence of inhibitors.

\subsection{Construction of $M$. smegmatis DprE1 point mutants}

M. smegmatis mutants were constructed using single-stranded DNA recombineering as described previously [34]. Briefly, electrocompetent $M$. smegmatis pJV53H (a version of pJV53 [35] with kanamycin resistance replaced by hygromycin resistance) were electroporated with a mixture of $100 \mathrm{ng}$ of a rpsL targeting oligonucleotide carrying a streptomycin-resistant mutation and $500 \mathrm{ng}$ of the oligo carrying one of the mutations in dprE1 (Table S2). Following a four-hour recovery phase, bacteria were plated onto 7H10-OADC plates with $20 \mu \mathrm{g} / \mathrm{mL}$ streptomycin and incubated for 5 days. Colonies were screened for the presence of the point mutation by colony PCR followed by digestion of the PCR products with Kpn2I (ThermoFisher Scientific) for one hour. Because of the biological basis of recombineering, positive colonies are indeed a mixture of bacteria harboring the wild-type and the mutant dprE1 alleles. Thus, colonies showing the undigested PCR product and the Kpn2I digestion products (Figure S3) were streaked onto agar plates, and colonies re-screened to eventually isolate the DprE1 mutants.

\subsection{Analysis of mycobacterial lipids using ${ }^{14} \mathrm{C}$ metabolic labeling}

M. tuberculosis H37Rv cells were grown in 7H9 broth supplemented with $10 \%$ albumin-dextrose-catalase and $0.025 \%$ Tyloxapol at $37^{\circ} \mathrm{C}$. Then, 100 ol culture aliquots $\left(\mathrm{OD}_{600}=0.32\right)$ were added into Eppendorf tubes containing 2 ol of DMSO or 2 @l of selamectin stock solution. Final concentrations of selamectin in the cultures were $0,4,8$ and $20 \mathrm{\odot g} / \mathrm{mL} .{ }^{14} \mathrm{C}$ acetate (specific activity $110 \mathrm{mCi} / \mathrm{mmol}, \mathrm{ARC}$ ) was added to each culture (final concentration, $0.5 \odot \mathrm{Ci} / \mathrm{mL}$ ) and cells cultivated for additional $24 \mathrm{~h}$.

M. smegmatis $\mathrm{mc}^{2} 155$ cells were grown in $200 \mu \mathrm{L}$ aliquots of $7 \mathrm{H} 9$ broth supplemented with $10 \%$ albumin-dextrose-catalase and $0.025 \%$ Tyloxapol at $37{ }^{\circ} \mathrm{C}$. Selamectin was added during the culture inoculation at a final concentration of $0,4,8$, 16 and $40 \odot \mathrm{g} / \mathrm{mL}$. After $43 \mathrm{~h}$ of cultivation in 96 -well plates, ${ }^{14} \mathrm{C}$ acetate was added to each culture (final concentration, $0.2 \odot \mathrm{Ci} / \mathrm{mL}$ ) and cells were cultivated for additional 6 h.

Whole cultures $(100 \mu \mathrm{L}$ or $200 \mu \mathrm{L})$ were transferred into $1.5 \mathrm{~mL}$ or $3 \mathrm{~mL}$ of chloroform/methanol (2:1) and lipids were extracted at $65{ }^{\circ} \mathrm{C}$ for 2-3 h. Samples were then subjected to biphasic Folch wash [36] (2×). Isolated lipids were dissolved in $30 \mu \mathrm{L}$ 
bioRxiv preprint doi: https://doi.org/10.1101/2021.12.04.471217; this version posted December 4, 2021. The copyright holder for this preprint

(which was not certified by peer review) is the author/funder, who has granted bioRxiv a license to display the preprint in perpetuity. It is made available under aCC-BY-NC-ND 4.0 International license.

of chloroform:methanol (2:1, v/v) and $5 \mu \mathrm{L}$ loaded on thin-layer chromatography (TLC) silica gel plates $\mathrm{F}_{254}$ (Merck). Lipids were separated in chloroform/methanol/water (20:4:0.5, v/v/v) and visualized using an Amersham ${ }^{\mathrm{TM}}$ Typhoon ${ }^{\mathrm{TM}}$ Biomolecular Imager or by autoradiography. 
Supplementary materials: Table S1: Oligonucleotides used for DprE1 mutagenesis; Table S2: Oligonucleotides used for M. smegmatis recombineering; Figure S1: Comparison of the binding sites occupied by the co-crystallized QN129 (thick tube representation, color by element + green carbons) and the docked selamectin; Figure S2: Sequence alignment of mycobacterial DprE1; Figure S3: Screening of M. smegmatis recombinants for point mutations; Figure S4: Time-kill kinetics of $M$. smegmatis DprE1 point mutants.

Author Contributions: Conceptualization: J.M.E-A, J.K, F.M, M.R.P, L.R.C and S.R-G. Experimental: J.M.E-A (genetic and phenotypic assays), G.D (phenotypic assays), H.G (lipid analysis), G.S (molecular and enzymatic assays), J.C.S (molecular and enzymatic assays), P.G (molecular modelling), F.M (molecular modelling), L.R.C (molecular and enzymatic assays). Writing - original draft: J.M.E-A, J.K, F.M, L.R.C and S.R-G. Writing - review \& editing: J.M.E-A, G.D, H.G, J.K, F.M, M.R.P, L.R.C and S.R-G.

Funding: This research was funded by a fellowship from the Spanish Government (Programa de Formación de Profesorado Universitario) Ref. FPU18/03873 to J.M.E-A, a grant from Slovak Research and Development Agency (n. APVV-19-0189) to J.K., a grant from Italian Ministry of University and Research (Dipartimenti di Eccellenza Program, 2018-2022) to Department of Biology and Biotechnology "L. Spallanzani", University of Pavia (G.D., G.S., M.R.P., and L.R.C.), and to Department of Biotechnology, Chemistry and Pharmacy, University of Siena (P.G. and F.M.).

Acknowledgments: We thank Katarína Mikušová from the Comenius University in Bratislava for helpful discussions during the performance of this work.

Conflicts of Interest: None to declare.

\section{References}

1. World Health Organization. Global Tuberculosis Report; 2020. https://apps.who.int/iris/rest/bitstreams/1312164/retrieve

2. Conradie, F.; Diacon, A. H.; Ngubane, N.; Howell, P.; Everitt, D.; Crook, A. M.; Mendel, C. M.; Egizi, E.; Moreira, J.; Timm, J.; McHugh, T. D.; Wills, G.; Bateson, A.; Hunt, R.; Van Niekerk, C.; Li, M.; Olugbosi, M.; Spigelman, M. Bedaquiline, Pretomanid and Linezolid for Treatment of Extensively Drug Resistant, Intolerant or Non-Responsive Multidrug Resistant Pulmonary Tuberculosis. N Engl J Med 2020, 382 (10), 893-902. https://doi.org/10.1056/NEJMoa1901814.

3. Dorman, S. E.; Nahid, P.; Kurbatova, E. V.; Goldberg, S. V.; Bozeman, L.; Burman, W. J.; Chang, K.-C.; Chen, M.; Cotton, M.; Dooley, K. E.; Engle, M.; Feng, P.-J.; Fletcher, C. V.; Ha, P.; Heilig, C. M.; Johnson, J. L.; Lessem, E.; Metchock, B.; Miro, J. M.; Nhung, N. V.; Pettit, A. C.; Phillips, P. P. J.; Podany, A. T.; Purfield, A. E.; Robergeau, K.; Samaneka, W.; Scott, N. A.; Sizemore, E.; Vernon, A.; Weiner, M.; Swindells, S.; Chaisson, R. E.; AIDS Clinical Trials Group and the Tuberculosis Trials Consortium. High-Dose Rifapentine with or without Moxifloxacin for Shortening Treatment of Pulmonary Tuberculosis: Study Protocol for TBTC Study 31/ACTG A5349 Phase 3 
Clinical Trial. Contemp Clin Trials 2020, 90, 105938. https://doi.org/10.1016/j.cct.2020.105938.

4. Pipeline I Working Group for New TB Drugs https://www.newtbdrugs.org/pipeline/clinical (accessed 2021 -07 -05).

5. World Health Organization, W. H.; Global Tuberculosis Programme. WHO Treatment Guidelines for Drug-Resistant Tuberculosis: 2016 Update. Who 2016, No. October, 56. https://apps. who.int/iris/rest/bitstreams/1061087/retrieve?

6. World Health Organization. Prioritization of pathogens to guide discovery, research and development of new antibiotics for drug-resistant bacterial infections including tuberculosis. Geneva; 2017. https://apps.who.int/iris/handle/10665/311820

7. World Health Organization. 2020 Antibacterial Agents in Clinical and Preclinical Development: An Overview and 2021. https://apps. who.int/iris/rest/bitstreams/1341746/retrieve

8. Farha, M. A.; Brown, E. D. Drug Repurposing for Antimicrobial Discovery. Nature Microbiology 2019, 4 (4), 565-577. https://doi.org/10.1038/s41564-019-0357-1.

9. Lim, L. E.; Vilchèze, C.; Ng, C.; Jacobs, W. R.; Ramón-García, S.; Thompson, C. J. Anthelmintic Avermectins Kill Mycobacterium tuberculosis, Including MultidrugResistant Clinical Strains. Antimicrobial Agents and Chemotherapy 2013, 57 (2), 1040 1046. https://doi.org/10.1128/AAC.01696-12.

10. Scherr, N.; Pluschke, G.; Thompson, C. J.; Ramón-García, S. Selamectin Is the Avermectin with the Best Potential for Buruli Ulcer Treatment. PLoS Neglected Tropical Diseases 2015, 9 (8), 1-10. https://doi.org/10.1371/journal.pntd.0003996.

11. Muñoz-Muñoz, L.; Shoen, C.; Sweet, G.; Vitoria, A.; Bull, T. J.; Cynamon, M.; Thompson, C. J.; Ramón-García, S. Repurposing Avermectins and Milbemycins against Mycobacteroides Abscessus and Other Nontuberculous Mycobacteria. Antibiotics 2021, 10 (4), 381. https://doi.org/10.3390/antibiotics10040381.

12. Burg, R. W.; Miller, B. M.; Baker, E. E.; Birnbaum, J.; Currie, S. A.; Hartman, R.; Kong, Y.-L.; Monaghan, R. L.; Olson, G.; Putter, I.; Tunac, J. B.; Wallick, H.; Stapley, E. O.; Oiwa, R.; Ōmura, S. Avermectins, New Family of Potent Anthelmintic Agents: Producing Organism and Fermentation. Antimicrob Agents Chemother 1979, 15 (3), 361367. https://doi.org/10.1128/AAC.15.3.361.

13. Ōmura, S. A Splendid Gift from the Earth: The Origins and Impact of the Avermectins (Nobel Lecture). Angewandte Chemie - International Edition 2016, 55 (35), 10190-10209. https://doi.org/10.1002/anie.201602164.

14. The Ivermectin Roadmappers; Billingsley, P.; Binka, F.; Chaccour, C.; Foy, B.; Gold, S.; Gonzalez-Silva, M.; Jacobson, J.; Jagoe, G.; Jones, C.; Kachur, P.; Kobylinski, K.; Last, A.; Lavery, J. V.; Mabey, D.; Mboera, D.; Mbogo, C.; Mendez-Lopez, A.; Rabinovich, N. R.; Rees, S.; Richards, F.; Rist, C.; Rockwood, J.; Ruiz-Castillo, P.; Sattabongkot, J.; Saute, F.; Slater, H.; Steer, A.; Xia, K.; Zullinger, R. A Roadmap for the Development of Ivermectin as a Complementary Malaria Vector Control Tool. Am J Trop Med Hyg 2020, 102 (2s), 3-24. https://doi.org/10.4269/ajtmh.19-0620.

15. Nolan, T. J.; Lok, J. B. Macrocyclic Lactones in the Treatment and Control of Parasitism in Small Companion Animals. Current Pharmaceutical Biotechnology 2012, 13 (6), 1078 1094. https://doi.org/10.2174/138920112800399167

16. Revolution. Prescribing Information. https://www.zoetisus.com/_locale-assets/mcmportal-assets/products/pdf/revolution-prescribing-information.pdf (Accessed July 12, 2021).

17. Wolstenholme, A.J.; Rogers, A.T. Glutamate-gated chloride channels and the mode of action of the avermectin/milbemycin anthelmintics. Parasitology 2005, 131 Suppl, S8595, doi:10.1017/S0031182005008218.

18. Mikusová, K.; Huang, H.; Yagi, T.; Holsters, M.; Vereecke, D.; D’Haeze, W.; Scherman, M. S.; Brennan, P. J.; McNeil, M. R.; Crick, D. C. Decaprenylphosphoryl 
Arabinofuranose, the Donor of the D-Arabinofuranosyl Residues of Mycobacterial Arabinan, Is Formed via a Two-Step Epimerization of Decaprenylphosphoryl Ribose. $J$ Bacteriol 2005, 187 (23), 8020-8025. https://doi.org/10.1128/JB.187.23.8020-8025.2005.

19. Abrahams, K. A.; Besra, G. S. Mycobacterial Cell Wall Biosynthesis: A Multifaceted

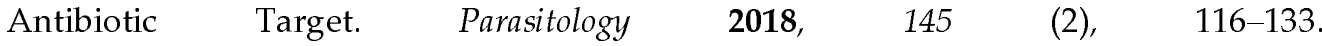
https://doi.org/10.1017/S0031182016002377.

20. Kolly, G. S.; Boldrin, F.; Sala, C.; Dhar, N.; Hartkoorn, R. C.; Ventura, M.; Serafini, A.; McKinney, J. D.; Manganelli, R.; Cole, S. T. Assessing the essentiality of the decaprenyl-phospho-d-arabinofuranose pathway in Mycobacterium tuberculosis using conditional mutants. Molecular microbiology 2014, 92 (1), 194-211. https://doi.org/10.1111/mmi.12546.

21. Degiacomi, G.; Belardinelli, J. M.; Pasca, M. R.; De Rossi, E.; Riccardi, G.; Chiarelli, L. R. Promiscuous Targets for Antitubercular Drug Discovery: The Paradigm of DprE1 and MmpL3. Applied Sciences 2020, 10 (2), 623. https://doi.org/10.3390/app10020623.

22. Makarov, V.; Manina, G.; Mikusova, K.; Möllmann, U.; Ryabova, O.; Saint-Joanis, B.; Dhar, N.; Pasca, M. R.; Buroni, S.; Lucarelli, A. P.; Milano, A.; De Rossi, E.; Belanova, M.; Bobovska, A.; Dianiskova, P.; Kordulakova, J.; Sala, C.; Fullam, E.; Schneider, P.; McKinney, J. D.; Brodin, P.; Christophe, T.; Waddell, S.; Butcher, P.; Albrethsen, J.; Rosenkrands, I.; Brosch, R.; Nandi, V.; Bharath, S.; Gaonkar, S.; Shandil, R. K.; Balasubramanian, V.; Balganesh, T.; Tyagi, S.; Grosset, J.; Riccardi, G.; Cole, S. T. Benzothiazinones Kill Mycobacterium tuberculosis by Blocking Arabinan Synthesis. Science 2009, 324 (5928), 801-804. https://doi.org/10.1126/science.1171583.

23. Neres, J.; Pojer, F.; Molteni, E.; Chiarelli, L. R.; Dhar, N.; Boy-Rottger, S.; Buroni, S.; Fullam, E.; Degiacomi, G.; Lucarelli, A. P.; Read, R. J.; Zanoni, G.; Edmondson, D. E.; De Rossi, E.; Pasca, M. R.; McKinney, J. D.; Dyson, P. J.; Riccardi, G.; Mattevi, A.; Cole, S. T.; Binda, C. Structural Basis for Benzothiazinone-Mediated Killing of Mycobacterium tuberculosis. Science Translational Medicine 2012, 4 (150), 150ra121-150ra121. https://doi.org/10.1126/scitranslmed.3004395.

24. Naik M, Humnabadkar V, Tantry SJ, Panda M, Narayan A, Guptha S, Panduga V, Manjrekar P, Jena LK, Koushik K, Shanbhag G, Jatheendranath S, Manjunatha MR, Gorai G, Bathula C, Rudrapatna S, Achar V, Sharma S, Ambady A, Hegde N, Mahadevaswamy J, Kaur P, Sambandamurthy VK, Awasthy D, Narayan C, Ravishankar S, Madhavapeddi P, Reddy J, Prabhakar K, Saralaya R, Chatterji M, Whiteaker J, McLaughlin B, Chiarelli LR, Riccardi G, Pasca MR, Binda C, Neres J, Dhar N, Signorino-Gelo F, McKinney JD, Ramachandran V, Shandil R, Tommasi R, Iyer PS, Narayanan S, Hosagrahara V, Kavanagh S, Dinesh N, Ghorpade SR. 4-aminoquinolone piperidine amides: noncovalent inhibitors of DprE1 with long residence time and potent antimycobacterial activity. J Med Chem. 2014 Jun 26;57(12):5419-34. doi: 10.1021/jm5005978.

25. Neres, J.; Hartkoorn, R. C.; Chiarelli, L. R.; Gadupudi, R.; Pasca, M. R.; Mori, G.; Venturelli, A.; Savina, S.; Makarov, V.; Kolly, G. S.; Molteni, E.; Binda, C.; Dhar, N.; Ferrari, S.; Brodin, P.; Delorme, V.; Landry, V.; de Jesus Lopes Ribeiro, A. L.; Farina, D.; Saxena, P.; Pojer, F.; Carta, A.; Luciani, R.; Porta, A.; Zanoni, G.; De Rossi, E.; Costi, M. P.; Riccardi, G.; Cole, S. T. 2-Carboxyquinoxalines Kill Mycobacterium tuberculosis through Noncovalent Inhibition of DprE1. ACS Chem Biol 2015, 10 (3), 705-714. https://doi.org/10.1021/cb5007163.

26. Makarov V, Neres J, Hartkoorn RC, Ryabova OB, Kazakova E, Šarkan M, Huszár S, Piton J, Kolly GS, Vocat A, Conroy TM, Mikušová K, Cole ST. The 8-PyrroleBenzothiazinones Are Noncovalent Inhibitors of DprE1 from Mycobacterium tuberculosis. Antimicrob Agents Chemother. 2015 Aug;59(8):4446-52. doi: 10.1128/AAC.00778-15 
27. Wolstenholme, A. J.; Rogers, A. T. Glutamate-Gated Chloride Channels and the Mode of Action of the Avermectin/Milbemycin Anthelmintics. Parasitology 2006, 131 (S1), S85-S95. https://doi.org/10.1017/s0031182005008218.

28. Karabanovich, G.; Dušek, J.; Savková, K.; Pavliš, O.; Pávková, I.; Korábečný, J.; Kučera, T.; Kočová Vlčková, H.; Huszár, S.; Konyariková, Z.; Konečná, K.; Jand’ourek, O.; Stolaříková, J.; Korduláková, J.; Vávrová, K.; Pávek, P.; Klimešová, V.; Hrabálek, A.; Mikušová, K.; Roh, J. Development of 3,5-Dinitrophenyl-Containing 1,2,4-Triazoles and Their Trifluoromethyl Analogues as Highly Efficient Antitubercular Agents Inhibiting Decaprenyl phosphoryl- $\beta$ - D -Ribofuranose 2'-Oxidase. J. Med. Chem. 2019, 62 (17), 8115-8139. https://doi.org/10.1021/acs.jmedchem.9b00912.

29. El-Saber Batiha, G.; Alqahtani, A.; Ilesanmi, O. B.; Saati, A. A.; El-Mleeh, A.; Hetta, H. F.; Magdy Beshbishy, A. Avermectin Derivatives, Pharmacokinetics, Therapeutic and Toxic Dosages, Mechanism of Action, and Their Biological Effects. Pharmaceuticals 2020, 13 (8), 196. https://doi.org/10.3390/ph13080196.

30. Stelitano, G.; Sammartino, J. C.; Chiarelli, L. R. Multitargeting Compounds: A Promising Strategy to Overcome Multi-Drug Resistant Tuberculosis. Molecules 2020, 25 (5), 1239. https://doi.org/10.3390/molecules25051239.

31. Maestro; Schrödinger, LLC: New York, NY, 2019.

32. Friesner, R. A.; Banks, J. L.; Murphy, R. B.; Halgren, T. A.; Klicic, J. J.; Mainz, D. T.; Repasky, M. P.; Knoll, E. H.; Shelley, M.; Perry, J. K.; Shaw, D. E.; Francis, P.; Shenkin, P. S. Glide: A New Approach for Rapid, Accurate Docking and Scoring. 1. Method and Assessment of Docking Accuracy. J Med Chem 2004, 47 (7), 1739-1749. https://doi.org/10.1021/jm0306430.

33. Bowers, K. J.; Chow, E.; Xu, H.; Dror, R. O.; Eastwood, M. P.; Gregersen, B. A.; Klepeis, J. L.; Kolossvary, I.; Moraes, M. A.; Sacerdoti, F. D.; Salmon, J. K.; Shan, Y.; Shaw, D. E. Scalable Algorithms for Molecular Dynamics Simulations on Commodity Clusters. In In SC '06: Proceedings of the 2006 ACM/IEEE Conference on Supercomputing; ACM Press, 2006.

34. Kessel, J. C. V.; Hatfull, G. F. Efficient Point Mutagenesis in Mycobacteria Using Single-Stranded DNA Recombineering: Characterization of Antimycobacterial Drug Targets. Molecular Microbiology 2008, 67 (5), 1094-1107. https://doi.org/10.1111/j.13652958.2008.06109.x.

35. Kessel, J. C. V.; Hatfull, G. F. Recombineering in Mycobacterium tuberculosis. Nat Methods 2007, 4 (2), 147-152. https://doi.org/10.1038/nmeth996.

36. Folch, J.; Lees, M.; Sloane Stanley, G. H. A Simple Method for the Isolation and Purification of Total Lipides from Animal Tissues. J Biol Chem 1957, 226 (1), 497-509. https://doi.org/10.1016/S0021-9258(18)64849-5.

37. Mori, G.; Orena, B. S.; Chiarelli, L. R.; Degiacomi, G.; Riabova, O.; Sammartino, J. C.; Makarov, V.; Riccardi, G.; Pasca, M. R. Rv0579 Is Involved in the Resistance to the TP053 Antitubercular Prodrug. Front. Microbiol. 2020, 11. https://doi.org/10.3389/fmicb.2020.00292.

38. Poce, G.; Bates, R. H.; Alfonso, S.; Cocozza, M.; Porretta, G. C.; Ballell, L.; Rullas, J.; Ortega, F.; De Logu, A.; Agus, E.; La Rosa, V.; Pasca, M. R.; De Rossi, E.; Wae, B.; Franzblau, S. G.; Manetti, F.; Botta, M.; Biava, M. Improved BM212 MmpL3 Inhibitor Analogue Shows Efficacy in Acute Murine Model of Tuberculosis Infection. PLoS One 2013, 8 (2), e56980. https://doi.org/10.1371/journal.pone.0056980.

39. Mori, G.; Chiarelli, L. R.; Esposito, M.; Makarov, V.; Bellinzoni, M.; Hartkoorn, R. C.; Degiacomi, G.; Boldrin, F.; Ekins, S.; de Jesus Lopes Ribeiro, A. L.; Marino, L. B.; Centárová, I.; Svetlíková, Z.; Blaško, J.; Kazakova, E.; Lepioshkin, A.; Barilone, N.; Zanoni, G.; Porta, A.; Fondi, M.; Fani, R.; Baulard, A. R.; Mikušová, K.; Alzari, P. M.; Manganelli, R.; de Carvalho, L. P. S.; Riccardi, G.; Cole, S. T.; Pasca, M. R. Thiophenecarboxamide Derivatives Activated by EthA Kill Mycobacterium tuberculosis 
by Inhibiting the CTP Synthetase PyrG. Chem Biol 2015, 22 (7), 917-927. https://doi.org/10.1016/j.chembiol.2015.05.016.

40. Degiacomi, G.; Sammartino, J. C.; Sinigiani, V.; Marra, P.; Urbani, A.; Pasca, M. R. In Vitro Study of Bedaquiline Resistance in Mycobacterium tuberculosis Multi-Drug Resistant Clinical Isolates. Front Microbiol 2020, 11, 559469. https://doi.org/10.3389/fmicb.2020.559469. 
Table 1. MIC of BTZ043 and selamectin against a set of $M$. tuberculosis mutants resistant to a variety of anti-TB compounds. Isoniazid was included as internal control of activity. The "references" column indicates the first reports for the strains used in this study. INH, isoniazid; STR, streptomycin; RIF, rifampicin; EMB, ethambutol; PYR, pyridomycin; ETH, ethionamide; CAP, capreomycin; BTZ, benzothiazinones; nd: not determined

\begin{tabular}{|c|c|c|c|c|c|}
\hline M. tuberculosis strain & $\begin{array}{c}\text { Strain resistance } \\
\text { profile }\end{array}$ & $\begin{array}{c}\text { MIC INH } \\
(\mu \mathrm{g} / \mathrm{mL})\end{array}$ & $\begin{array}{c}\text { MIC SEL } \\
(\mu \mathrm{g} / \mathrm{mL})\end{array}$ & $\begin{array}{c}\text { MIC } \\
\text { BTZ043 } \\
\text { (ng/mL) }\end{array}$ & References \\
\hline $\mathrm{H} 37 \mathrm{Rv}$ & & 0.025 & 4 & 1 & \\
\hline H37Rv-pSODIT & & 0.025 & 4 & 1 & \\
\hline 53.3 (Rv2466c, W28S) & ТР053 & 0.025 & $2-4$ & nd & [37] \\
\hline 53.8 (Rv0579, L240V) & ТР053 & 0.025 & $2-4$ & nd & [37] \\
\hline NTB1 (DprE1 C387S) & BTZ & 0.025 & 2 & $>1000$ & [22] \\
\hline NTB9 (DprE1 C387G & BTZ & 0.025 & 2 & 1000 & [22] \\
\hline H37Rv-pSODIT:d prE1 & BTZ & 0.025 & 4 & 1000 & \\
\hline DR1 (mmpL3, V681I) & 1,5-diarylpyrroles & 0.025 & $2-4$ & nd & [38] \\
\hline Ty1 (Rv3405c, c190t) & Ту $38 \mathrm{c}$ & 0.025 & $2-4$ & nd & [26] \\
\hline 88.1 (coaA, Q207R) & $\begin{array}{c}\text { Thiophenecarboxamide } \\
\text { derivatives }\end{array}$ & 0.025 & $2-4$ & nd & {$[39]$} \\
\hline 88.7 (pyrG, V186G) & $\begin{array}{c}\text { Thiophenecarboxamide } \\
\text { derivatives }\end{array}$ & 0.025 & $2-4$ & nd & [39] \\
\hline 81.10 (ethA, D1109-37) & $\begin{array}{c}\text { Thiophenecarboxamide } \\
\text { derivatives }\end{array}$ & 0.025 & $4-8$ & nd & [39] \\
\hline IC1 & STR, INH, RIF, EMB & $>0.2$ & $4-8$ & nd & [40] \\
\hline IC2 & $\begin{array}{l}\text { STR, INH, RIF, EMB, } \\
\text { PYR, ETH, CAP }\end{array}$ & $>0.2$ & $4-8$ & nd & [40] \\
\hline
\end{tabular}


bioRxiv preprint doi: https://doi.org/10.1101/2021.12.04.471217· this version posted December 4, 2021. The copyright holder for this preprint (which was not certified by peer review) is the author/funder, who has granted bioRxiv a license to display the preprint in perpetuity. It is made available under aCC-BY-NC-ND 4.0 International license.

Table 2. Enzymatic activity of selected avermectins against the purified $M$. smegmatis DprE1. 'BTZ data was obtained from reference [26]

\begin{tabular}{cc}
\hline Avermectin & IC50 $(\mu \mathrm{M})$ \\
\hline Ivermectin & $13.2 \pm 3.3$ \\
Milbemycin oxime & $25.5 \pm 5.6$ \\
Moxidectin & $6.1 \pm 0.9$ \\
Selamectin & $2.6 \pm 1.1$ \\
BTZ-043 $^{1}$ & 0.004 \\
\hline
\end{tabular}


Table 3. Steady state kinetic parameters of M. smegmatis DprE1 and IC 50 values of selamectin. SEL, selamectin; nd, not determined.

\begin{tabular}{lllll}
\hline $\begin{array}{l}\text { DprE1 } \\
\text { variant }\end{array}$ & $\begin{array}{l}K_{m} \\
(\mu \mathrm{M})\end{array}$ & $\begin{array}{l}k_{\text {cat }} \\
\left(\mathbf{m i n}^{-1}\right)\end{array}$ & $\begin{array}{l}k_{\text {cat }} / K_{m} \\
\left(\mathbf{m i n}^{-1} \boldsymbol{\mu M}-\mathbf{1}\right)\end{array}$ & $\begin{array}{l}\text { IC } 50 \text { SEL } \\
(\mu \mathrm{M})\end{array}$ \\
\hline Wild-type & $0.19 \pm 0.20$ & $5.1 \pm 0.4$ & $26.8 \pm 1.1$ & $2.6 \pm 1.1$ \\
C394A & $0.33 \pm 0.22$ & $2.8 \pm 0.5$ & $8.5 \pm 0.6$ & $3.9 \pm 1.0$ \\
C394G & nd & nd & nd & nd \\
C394S & $0.31 \pm 0.15$ & $1.6 \pm 0.3$ & $5.2 \pm 0.6$ & $12.1 \pm 1.2$ \\
L282F & $0.16 \pm 0.12$ & $4.5 \pm 0.3$ & $28.1 \pm 1.4$ & $1.0 \pm 0.7$ \\
L282V & $0.21 \pm 0.15$ & $4.3 \pm 0.4$ & $20.4 \pm 1.1$ & $50.6 \pm 9.2$ \\
\hline
\end{tabular}


bioRxiv preprint doi: https://doi.org/10.1101/2021.12 04.471217; this version posted December 4, 2021. The copyright holder for this preprint (which was not certified by peer review) is the author/funder, who has granted bioRxiv a license to display the preprint in perpetuity. It is made available under aCC-BY-NC-ND 4.0 International license.

Table 4. MIC of BTZ-043 and selamectin against M. smegmatis point mutants at the Leu282 residue. SEL, selamectin

\begin{tabular}{ccc}
\hline M. smegmatis strain & MIC BTZ-043 (ng/mL) & MIC SEL $(\mu \mathrm{g} / \mathbf{m L})$ \\
\hline $\mathrm{mc}^{2} 155$ & 8 & 4 \\
$\mathrm{mc}^{2} 155-$ pJV53H & 8 & 4 \\
$\mathrm{mc}^{2}$ 155-pJV53H DprE1 L282F & 8 & 4 \\
$\mathrm{mc}^{2}$ 155-pJV53H DprE1 L282V & 4 & 4 \\
\hline
\end{tabular}



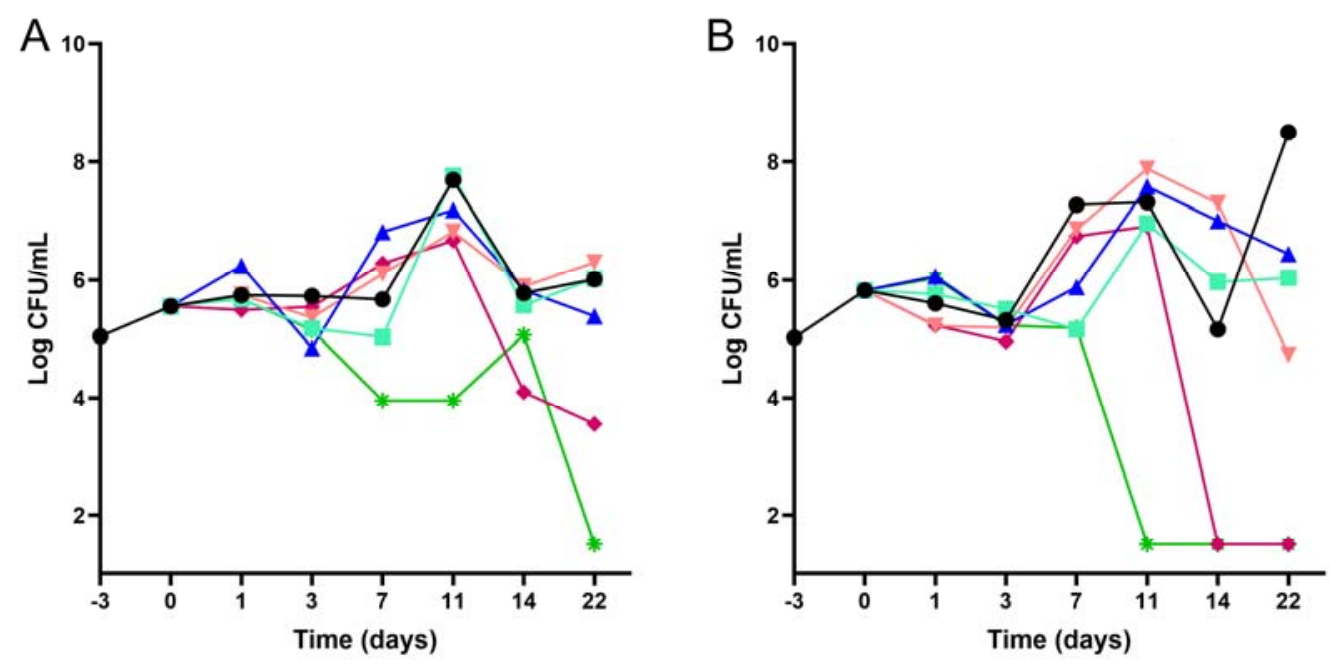

Figure 1. Time-kill kinetics of selamectin against wild-type M. tuberculosis H37Rv and NTB1 mutant strains. 22-day kill kinetic assays were performed using various concentrations of selamectin $(\bullet 0 \mu \mathrm{g} / \mathrm{mL} ; \square 0.1 \mu \mathrm{g} / \mathrm{mL} ; \boldsymbol{\Delta} 0.5 \mu \mathrm{g} / \mathrm{mL} ; \nabla 2 \mu \mathrm{g} / \mathrm{mL} ; \diamond 8$ $\mu \mathrm{g} / \mathrm{mL}$; $32 \mu \mathrm{g} / \mathrm{mL}$ ) against the reference M. tuberculosis H37Rv (panel A) and NTB1 (panel B) strains. 

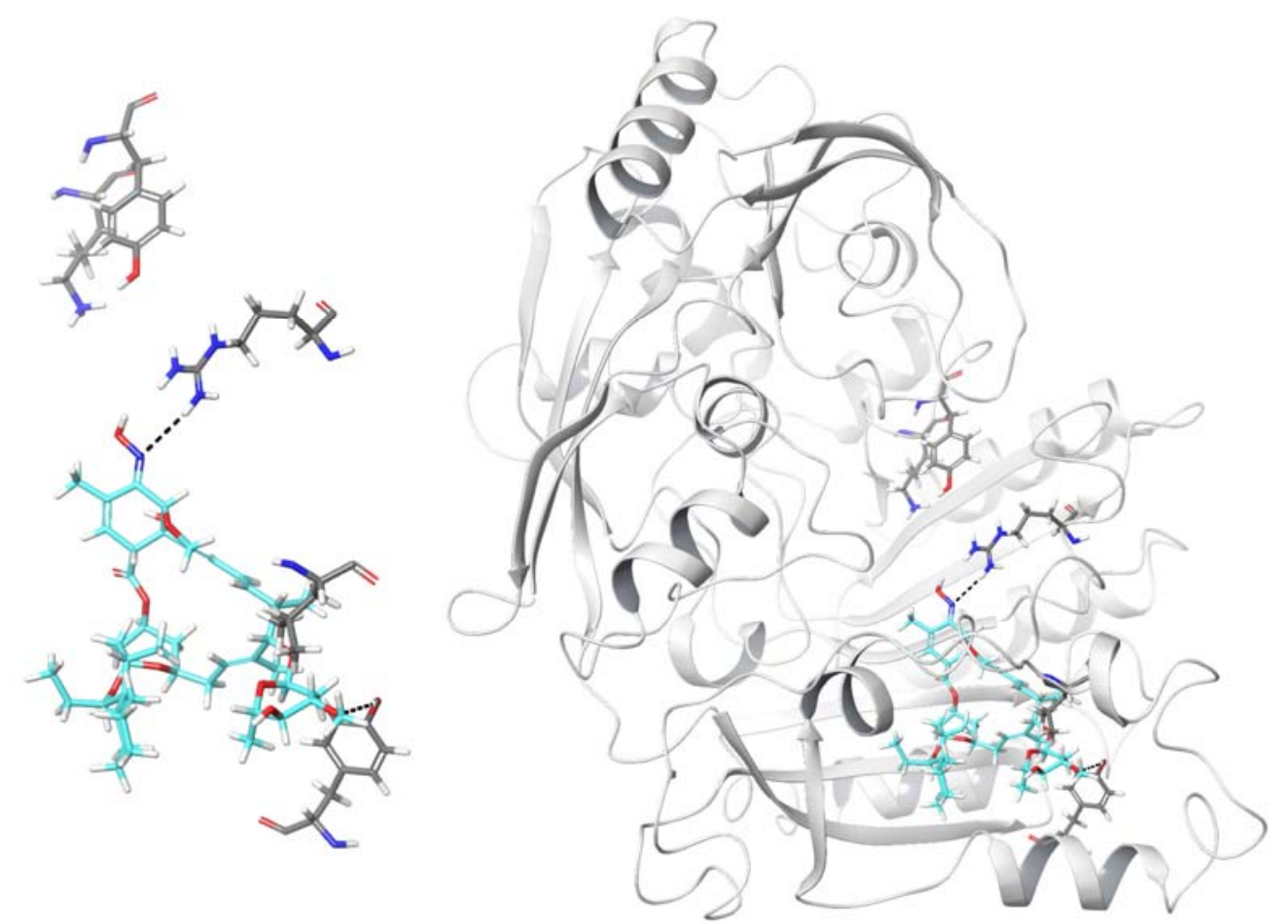

Figure 2. Graphical representation of one of the best-scored conformations of selamectin (thick tube representation, color by element + cyan carbons) within the DprE1 binding site (thick tube representation, left panel). For the sake of clarity, only a few amino acids are shown. Lys418, Tyr60, Arg325, Leu275, and Tyr297 (from left to right in the pictures) are represented by thick tubes. Hydrogen bonds that involve Arg325 (and the oxime nitrogen atom of selamectin) and Tyr297 (and the methoxy oxygen atom of selamectin) are shown by dashed black lines. The right panel shows the overall view of the selamectin/DprE1 docked complex. 
bioRxiv preprint doi: https://doi.org/10.1101/2021.12.04.471217· this version posted December 4, 2021. The copyright holder for this preprint (which was not certified by peer review) is the author/funder, who has granted bioRxiv a license to display the preprint in perpetuity. It is made available under aCC-BY-NC-ND 4.0 International license.

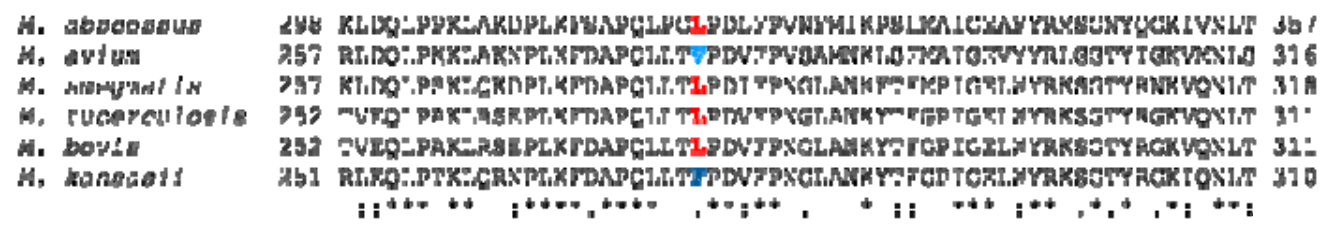

Figure 3. Sequence alignment of the DprE1 region around M. tuberculosis Leu275

(shown in red). Full sequence alignment is shown in Figure S2 
bioRxiv preprint doi: https://doi.org/10.1101/2021.12.04.471217; this version posted December 4, 2021. The copyright holder for this preprint (which was not certified by peer review) is the author/funder, who has granted bioRxiv a license to display the preprint in perpetuity. It is made available under aCC-BY-NC-ND 4.0 International license.

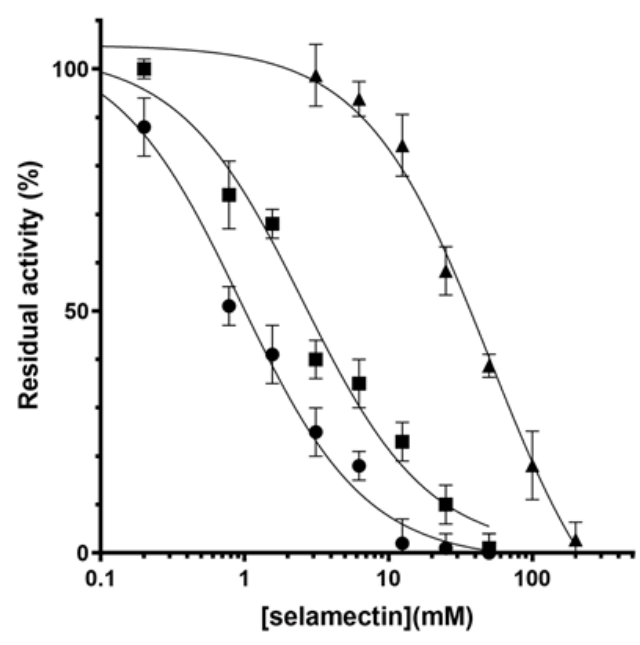

Figure 4. Dose response inhibition of DprE1 by selamectin. IC50 determination of selamectin against wild type $(\boldsymbol{\bullet}), \mathrm{L} 282 \mathrm{~F}(\bullet)$ and L282V ( $\mathbf{\Delta})$ M. smegmatis DprE1. 

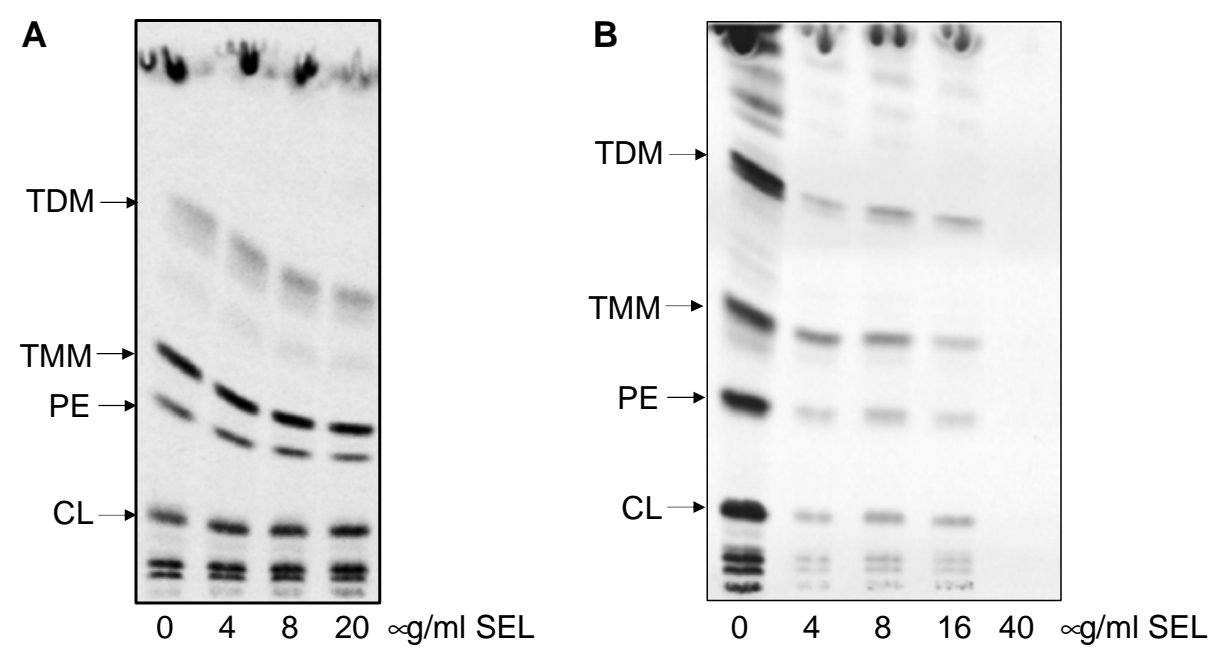

Figure 5. Analysis of mycobacterial lipid composition upon treatment with selamectin. Evaluation of the effect of selamectin (SEL) on the lipids of $M$. tuberculosis H37Rv (A) and $M$. smegmatis $\mathrm{mc}^{2} 155$ (B) metabolically labeled with ${ }^{14} \mathrm{C}$ acetate. Autoradiogram of TLC analysis of lipids separated in chloroform/methanol/water (20:4:0.5). TDM, trehalose dimycolates; TMM, trehalose monomycolates; PE, phosphatidylethanolamine; CL, cardiolipin; SEL, selamectin. 